\title{
Parameters sensitivity analysis for a crop growth model applied to winter wheat in the Huanghuaihai Plain in China
}

\author{
M. Liu ${ }^{1}$, B. $\mathrm{He}^{2}$, A. Lü̈ ${ }^{3}$ L. Zhou ${ }^{4}$, and J. Wu ${ }^{2}$ \\ ${ }^{1}$ National Disaster Reduction Center of China, Beijing, China \\ ${ }^{2}$ Beijing Normal University, Beijing, China \\ ${ }^{3}$ Institute of Geographic Sciences and Natural Resources Research, CAS, Beijing, China \\ ${ }^{4}$ China National Environmental Monitoring Center, Beijing, China \\ Received: 20 May 2014 - Accepted: 21 May 2014 - Published: 11 June 2014 \\ Correspondence to: A. Lü (kmsliu@gmail.com) \\ Published by Copernicus Publications on behalf of the European Geosciences Union.
}

\begin{abstract}
Parameters sensitivity analysis is a crucial step in effective model calibration. It quantitatively apportions the variation of model output to different sources of variation, and identifies how "sensitive" a model is to changes in the values of model parameters.

5 Through calibration of parameters that are sensitive to model outputs, parameter estimation becomes more efficient. Due to uncertainties associated with yield estimates in a regional assessment, field-based models that perform well at field scale are not accurate enough to model at regional scale. Conducting parameters sensitivity analysis at the regional scale and analyzing the differences of parameter sensitivity between

10 stations would make model calibration and validation in different sub-regions more efficient. Further, it would benefit the model applied to the regional scale. Through simulating $2000 \times 22$ samples for 10 stations in the Huanghuaihai Plain, this study discovered that TB (Optimal temperature), HI (Normal harvest index), WA (Potential radiation use efficiency), BN2 (Normal fraction of $\mathrm{N}$ in crop biomass at mid-season) and RWPC1

15 (Fraction of root weight at emergency) are more sensitive than other parameters. Parameters that determine nutrition supplement and LAI development have higher global sensitivity indices than first-order indices. For spatial application, soil diversity is crucial because soil is responsible for crop parameters sensitivity index differences between sites.
\end{abstract}

\section{Introduction}

Parameters sensitivity analysis is used to estimate the rate of change in model outputs and changes in parameters. It is used to qualitatively or quantitatively apportion the variation of model outputs to different sources and to identify which parameters most strongly affect the model outputs (Klepper, 1997; Saltelli et al., 1999; Xu et al., 2004; Green and Whittemore, 2005). Parameters sensitivity analysis is an important step in model localization and regionalizing (Wu et al., 2009), and is widely used in distributed 
hydrology models (Griensven et al., 2006), ecological models (Janse et al., 2010), environmental models (Norton, 2008) and crop simulation models (Lamboni et al., 2009). It is very useful for data preparation because of higher precision requirement of the sensitive parameters. It can also make model calibration more efficient by making calibration necessary for only the sensitive parameters (Makowski et al., 2006).

Local sensitivity analysis and global sensitivity analysis are two widely-used methods in parameter sensitivity analysis. Local sensitivity analysis concentrates on the main (first-order) impact of factors on the model (Pappenberger et al., 2008). The problem of parameter identifiability is commonly studied using local sensitivity analysis (Brun

10 et al., 2002). Local sensitivity analysis is well suited for linear and near-linear models because it only considers single parameter impact on the model output while ignoring the combined effects with other parameters (Varella et al., 2010). Global sensitivity analysis takes into account the entire range of variation of model inputs and aims to apportion uncertainty of the whole output to model input uncertainties (Xu and Gertner,

15 2007). Global sensitivity analysis is thus widely-used in non-linear models. Many global sensitivity analysis methods exist, including Morris, Sobol, EFAST (Extended Fourier Amplitude Sensitivity Test). In the Morris method, factors can be ranked in the order of their importance, but the percentage of output variation caused by each factor cannot be quantified (Saltelli et al., 1999; Pappenberger et al., 2008). Variance-based methods

20 such as the Sobol method and the EFAST method use model output variance as an indicator of parameter importance (Saltelli et al., 1999; Xu et al., 2004; Hasofer, 2009). Both methods require many model simulations, but do not rely on special assumptions about model behavior (Varella et al., 2010).

Dynamic crop models can be applied to evaluate the potential of crop produc25 tion, to improve water and fertility management, to forecast crop yield, and to evaluate the impact of climate change on crop growth. Most crop models such as EPIC, CERES, CropSyst, etc., which integrate the major physiological processes in the soil-crop-atmosphere-management systems, are complex non-linear dynamic models (Makowski et al., 2006). Each has many parameters in crop, soil, weather, and

3869

tillage management. The estimation of these parameters is important because crop model performances largely depend on accuracy of parameter estimation (Wallach et al., 2001; Varella et al., 2010). Methods of global sensitivity analysis allow for the full uncertainty range of the parameters to be explored and analyzed through a complex 5 model, and for their interactions to be estimated by varying them concurrently (Saltelli et al., 2000). The local sensitivity index reflects only the main effect of a given parameter on model output. However, the global sensitivity index reflects all of the effects of said parameter on model output, including interactions with other parameters. It is suitable for non-linear models like crop growth models.

10 Recently, sensitivity analysis on crop growth has focused on field scale analysis (Makowski et al., 2006; Varella et al., 2010). There is growing interest in forecasting crop production at the regional scale to represent the influence of climate change, environmental degradation and other regional factors on crop growth. Parameter sensitivity analysis at the regional scale and analyzing differences of parameter sensitivity between different sites, would allow for selection of the most sensitive parameters for model calibration. This would improve the accuracy of the model at the regional scale. This study aims to develop a method to analyze the crop parameters' sensitivity at the regional scale. The first-order and the global sensitivity indices of the crop parameters of the EPIC crop growth model were calculated in 10 sites in the Huanghuaihai Plain

20 of Northern China using the EFAST sensitivity method, analysis of the difference of first-order and global sensitivity indices, and the differences of sensitivity indices between different sites. This study would be useful for crop simulation models applied at the regional scale. 


\section{Methods}

\subsection{Extended FAST}

Crop simulation models are complex nonlinear dynamic models simulating output variables related to crop yield, crop quality. EFAST (Extended Fourier Amplitude Sensitiv-

5 ity Test) can obtain first-order sensitivity indices (local sensitivity indices) and global sensitivity indices concurrently by using the same sampling vectors. It performs a judicious deterministic sampling to explore the parameter space, allowing for the reduction of these integrals to one-dimensional integrals using Fourier decompositions (Varella et al., 2010).

EFAST is a global sensitivity method that is based on the analysis of model output variance (Saltelli et al., 2000). Total variance of the output caused by the selected parameters can be partitioned as:

$V=\sum_{i} V_{i}+\sum_{i \neq j} V_{i j}+\sum_{i \neq j \neq m} V_{i j m}+\cdots+\sum V_{12 \cdots k}$

Where $V$ is the total variance (the variance of the model output), $V_{i}$ is the local (or main) ${ }_{5}$ effect of the parameter $i$, and the other terms measure the interaction effect of many parameters.

The sensitivities of each parameter are defined by:

$S_{i}=\frac{V_{i}}{V}, \quad S_{i j}=\frac{V_{i j}}{V}, \quad S_{i j m}=\frac{V_{i j m}}{V}, \quad S=S_{i}+S_{i j}+S_{i j m}+\cdots+S_{12 \cdots k}$

where $S_{i}$ is the first-order (local) sensitivity index for the parameter $i$, the fraction of the 20 variance of the output is explained by parameter $i, S_{i j}$ is the second-order sensitivity index for the interaction of parameters $i$ and $j$, and $S$ is the total (global) sensitivity index for parameter $i$ (the sum of all effects (first and higher order) involving parameter i) (Makowski et al., 2006; Wu et al., 2009).

The first step in the EFAST process is sampling using the Monte Carlo method. In EFAST, the analyzed model was represented as $y=f(x)$. The sampling vectors of crop parameters were defined as:

$x_{i}=G_{i}\left\{\frac{1}{2}+\frac{1}{\pi} \arcsin \left[\sin \left(\omega_{i} s+\varphi_{i}\right)\right]\right\} \quad \forall i=1,2, \cdots, n$

5 where $s$ is a scalar variable varying over range $-\pi<s<+\pi, \omega_{i}$ are sets of integer angular frequencies, $\phi_{i}$ are random phase-shifts chosen uniformly in $[0,2 \pi)$, and $G_{i}$ is the transformation function. In the predetermined transformation equation, the frequency of change in the parameter analyzed is more than 8 times of said frequency in other parameters. It requires at least 65 samples for global sensitivity analysis on each 10 parameter (Saltelli et al., 2000).

The next step involves simulating crop growth using these parameter sets. The variance of model output and the variance made by each parameter are calculated by the spectrum of the Fourier series expansion. The model $f(x)$ can be expanded into Fourier series $g(s)$ :

$15 \quad y=g(s)=\sum_{p=-\infty}^{\infty}\left(A_{p} \cos p s+B_{p} \sin p s\right)$

Where the Fourier coefficients $A_{p}$ and $B_{p}$ are defined as:

$A_{p}=\frac{1}{2 \pi} \int_{-\pi}^{\pi} g(s) \cos p s \mathrm{~d} s \quad B_{p}=\frac{1}{2 \pi} \int_{-\pi}^{\pi} g(s) \sin p s \mathrm{~d} s$

The spectrum of the Fourier series expansion is defined as:

$\wedge_{p}=A_{p}^{2}+B_{p}^{2}$ 
Since $g(s)$ is a real-valued function, $A_{p}, B_{p}$ and $\wedge_{p}$ have the following properties: $A$ $p=A_{p}, B-p=B_{p}$, and $\wedge-p=\wedge_{p}$. By evaluating the spectrum for the fundamental frequency $\omega_{i}$ and its higher harmonics $p \omega_{i}$, we can estimate $V_{i}$, the portion of the output variance $V$ arising from the uncertainty of parameter $i$ :

${ }_{5} V_{i}=\sum_{p \in Z^{0}} \wedge_{p \omega_{i}}=2 \sum_{p=1}^{\infty} \wedge_{p \omega_{i}}$

By summing all the $\wedge_{p}$, the total variance is estimated as follows:

$V=\sum_{p \in Z^{0}} \wedge_{p}=2 \sum_{p=1}^{\infty} \wedge_{p}$

The ratio $V_{i} / V$ is the required estimate of the main effect of parameter $i$ on the model output (Makowski et al., 2006). Global sensitivity indices consider the influence of other

10 parameters except the one analyzed, and are calculated by subtracting the total spectrum on the frequency below half of that of the parameter analyzed from the total spectrum.

\subsection{The model description}

EPIC (Erosion Productivity Impact Calculator) was developed in the United States to in15 vestigate the relationship between erosion and soil productivity and was subsequently enhanced by the further addition of modules to improve the simulation of plant growth (Williams et al., 1989). The model was further extended to include environmental assessment of pesticides and water quality and renamed as the Environmental Policy Integrated Climate Model (Rinaldi, 2001). The model integrates the major processes

20 that occur in the soil-crop-atmosphere-management systems, uses a daily time-step to simulate weather, hydrology, soil erosion, nutrient cycling, tillage, crop management and field-scale costs and returns (Williams, 1995).

3873

EPIC uses a single model for simulating all crops. All crops have unique values for different genetic coefficients like potential radiation-use efficiency, and leaf area index (LAI) curve coefficients, etc. EPIC uses radiation-use efficiency to calculate photosynthetic production of biomass. Interception of solar radiation is estimated as a function 5 of LAI. And LAI is simulated with equations determined by heat units, the maximum LAI for the crop, some genetic coefficients that initiate LAI increase and decline, and five environmental stresses (water, temperature, nutrients (Nitrogen and Phosphorus), aeration and radiation) (Easterling et al., 1998). The potential biomass is adjusted daily through multiplication by the environmental stress index, which is the minimum of five environmental stress indices. The water stress index is the ratio of soil-available water to potential water demand of crop. The temperature stress index is computed using a function dependent upon the daily average temperature, the optimal temperature, and the base temperature for crop growth. The $\mathrm{N}$ and $\mathrm{P}$ stress indices are based on the ratio of accumulated plant $\mathrm{N}$ and $\mathrm{P}$ to the optimal values of $\mathrm{N}$ and $\mathrm{P}$. The aera15 tion stress index is estimated as a function of soil water relative to porosity in the root zone (Tan and Shibasaki, 2003). Atmospheric $\mathrm{CO}_{2}$ concentration also influences photosynthesis by increasing radiation-use efficiency. Crop yields are estimated by multiplying the above-ground biomass at maturity (determined by accumulation of heat units or specified harvest date) by a harvest index (economic yield divided by above20 ground biomass) as specified for the particular crop (Easterling et al., 1998). EPIC is well-suited for relative comparisons of soil, crops, and management scenarios and provides accurate estimation of field yields in the United States, France, China, and other countries (Bryant et al., 1992; Satya and Ryosuke, 2001; Kang et al., 2002; Tan and Shibasaki, 2003; Liu et al., 2007).

25 EPIC requires two data sets to function. One required data set is the observed data set, which includes basic geographic information about a station, weather data, soil data, and crop management information about irrigation and fertilization. Daily weather data includes the minimum and maximum air temperature, daily precipitation, relative humidity, solar radiation and wind speed. EPIC can accept up to 20 parameters for 
15 soil layers. However, only the following seven parameters are required for each soil layer: depth, percent sand, percent silt, bulk density, $\mathrm{pH}$, percent organic carbon, and percent calcium carbonate. Soil data in this study were obtained from the FAO (Food and Agriculture Organization of the United Nations) (Tan and Ryosuke, 2003; 5 Saxton and Rawls, 2004; Wang, 2008). EPIC requires agricultural management data on fertilization, tillage, planting, harvesting, and irrigation. The second data set consists of parameter files such as: the crop parameter file, including genetic coefficients of crop cultivars; the pesticide parameter file; and fertilizer parameter file, which is used to determine crop growth and yield.

\subsection{Study area}

The Huanghuaihai Plain, located in Northern China, is the second largest plain in China. It covers more than $380000 \mathrm{~km}^{2}$. The average annual precipitation in this area is $500-800 \mathrm{~mm}$, and $60-80 \%$ of this precipitation occurs between June and September. It is an important agricultural area in China. Winter wheat and summer corn are

15 the region's main crops. Winter wheat was chosen as the study crop for this study. The winter wheat growth season is from October to June.

\subsection{Steps for regional parameter sensitivity analysis based on EFAST}

To analyze global sensitivity of EPIC crop growth model output to the crop parameters in the Huanghuaihai Plain, we designed the experiment as follows:

20 1. Site Selection. There are the three major soil types in the Huanghuaihai Plain: cinnamon soil, fluvo-aquic soil, and brown soil. Cinnamon soil is distributed in the piedmont zone of the Taihang, Taishan, and Yanshan Mountains, where elevation is less than $500 \mathrm{~m}$ the underground water level is below $3 \mathrm{~m}$. Brown soil is distributed in the Wolds area of the Shandong peninsula. Most of the Huanghuaihai Plain is alluvium by the Yellow, Huai, and Hai Rivers, making fluvo-aquic soil dominant. In this study, 10 uniformly-distributed sites were chosen (Fig. 1 and 3875

Table 1). These sites may be classified into 3 groups according to the soil type: cinnamon soil: Shijiazhuang, Xingtai, Xinxiang; fluvo-aquic soil: Botou, Lingxian, Huimin, Shangqiu; and brown soil: Weihai, Qingdao, Rizhao.

2. Sensitivity analysis. 22 genetic coefficients of the EPIC crop growth model were selected for analysis (Table 2). As genetic coefficients vary across genotypes and regions, the estimation of these parameters must be based on specific measurements collected for each genotype (Makowski et al., 2006). Sensitivity analysis on these parameters can make model calibration and validation more efficient.

3. Sample generation. 2000 samples per parameter were generated using the Monte Carlo method. The value of parameter samples were scattered in a range of 0.8 to 1.2 times the initial value.

\section{Results and discussions}

The results of global sensitivity analysis of the 22 parameters in the selected 10 stations are shown in Tables 3 and 4 . We selected 2 sites per soil type to show the differences 15 between first-order and global sensitivity indices (Fig. 2).

\subsection{First-order sensitivity indices}

The first-order sensitivity indices reflected the main (local) effect of the parameter on the model output. The results are shown in the Table 3.

The first-order sensitivity indices of TB (Optimal temperature), $\mathrm{HI}$ (Normal harvest in20 dex), WA (Potential radiation use efficiency), BN2 (Normal fraction of $\mathrm{N}$ in crop biomass at mid-season) and RWPC1 (Fraction of root weight at emergency) were much higher than those of the other parameters. The highest first-order sensitivity index of TB was more than 0.57 in Linxian, meaning that $57 \%$ of the variation of the model output was determined by TB. However, the sensitivity indices of TB in Weihai, Qingdao and 
Rizhao differed from other stations, where the indices were less than 0.20 . $\mathrm{HI}$ was also a more sensitive parameter. These were 3 stations at which the global sensitivity indices of HI were the highest: Weihai, Qingdao and Rizhao. The indices are greater than 0.3 and the largest is in Qingdao, at slightly less than 0.70 . The first order sensitivity

5 indices only showed the direct influence of the parameter. Temperature was one important factor that determined crop growth and development, and this made TB a sensitive parameter. EPIC used a general plant growth model with crop specific parameters to simulate the growth of wheat, rice, maize, grain. WA was a factor that determined how much radiation could be absorbed by crop when water and nutrients unlimited. Crop

10 yield, the main output of the EPIC crop simulation model, was calculated by multiplying above-ground biomass by $\mathrm{HI}$. WA and $\mathrm{HI}$ were the parameters that affected biomass assimilation and yield. This made their first-order sensitivity indices much higher than those of other parameters.

\subsection{Global sensitivity indices}

15 Global sensitivity indices are shown in Table 4. The global sensitivity indices reflect the sum of all effects involving the said parameter, including interactions with all other parameters. TB (Optimal temperature), HI (Normal harvest index), WA (Potential radiation use efficiency), BN2 (Normal fraction of N in crop biomass at mid-season) and RWPC1 (Fraction of root weight at emergency) were the five most-sensitive parameters.

20 According to global sensitivity indices, TB was the most sensitive factor in seven of the ten selected stations. The highest index was 0.66 in Linxian, meaning that $66 \%$ of the variation of the model output was influenced by TB. As shown in Table 3, the first order sensitivity index of TB in Linxian was about 0.57 . In this case, approximately $9 \%$ of the variation of the model output was controlled by the interaction of TB with other 25 parameters. $\mathrm{HI}$ is also a highly-sensitive parameter. There were 3 stations in which the global sensitivity index of $\mathrm{HI}$ was the highest. The highest of these indices was 0.72 in Qingtao, and the lowest was approximately 0.16 in Botou. BN2 is the most sensitive parameters among fertilizer parameters (BN1, BN2, BN3, BP1, BP2, and BP3). This 3877

was similar to the result of the first-order sensitivity indices. The global sensitivity index of the analyzed parameter is calculated as the sum of output variations related to the said parameter. Thus, it includes the first-order sensitivity index and the interaction with other parameters. TB, $\mathrm{HI}$ and WA were most sensitive parameters in first-order 5 sensitive analysis. The interaction with other parameters was usually much less than the first-order interaction in most analyzed parameters. Thus these three parameters became more sensitive.

\subsection{The difference between the first-order and global sensitivity index}

Figure 2 shows the differences between first-order and global sensitivity indices in 6 10 selected stations, which may be divided into 3 categories according to soil type.

The global sensitivity indices of the parameters describing the necessary nutrients and LAI development were much greater than their first-order sensitivity indices. Plant growth is constrained by environmental stresses, including water, nutrients and temperature. So the potential biomass is adjusted daily by multiplying it by the minimum 15 of these environmental stresses in EPIC. The water-stress factor is computed by considering water supply and demand. Temperature stress is computed using a function dependent upon the daily average temperature, the optimal temperature, and the base temperature for the crop. The $\mathrm{N}$ and $\mathrm{P}$ stress factors are based on the ratio of accumulated plant $N$ and $P$ to the optimal value of $N$ and $P$ (Tan and Shibasaki, 2003).

20 The $\mathrm{HI}$ also varies with water stress, temperature stress and nutrition (N, P) stress. So the parameters describing environmental factors, such as BP1, BN2, and BN3, had global sensitivity indices, that were much larger than their first-order sensitivity indices. LAI played an important role in calculation of solar energy absorbed by the plant. In EPIC, interception of solar radiation is estimated as a function of crop LAI. The DMLA 25 parameter determined the maximum potential LAI. Parameters DLP1 and DLP2 determined the point in the growing season when leaf area increased rapidly, and when the rate of increase for leaf area smoothed. DLAl determined the point in the growing season when leaf area declined due to leaf senescence. The parameters describing 
LAI increment and decline also affect the biomass assimilation and yield indirectly. The global sensitivity indices of these parameters were much larger than their first-order sensitivity indices.

\subsection{The difference between sites}

5 Global sensitivity indices of the same crop parameter differ from station to station (Tables 3 and 4; Fig. 2). We selected Shijiazhang and Xinxiang from the sites with cinnamon soil, Botou and Shangqiu from the sites with fluvo-aquic soil, and Weihai and Rizhao from the sites with brown soil. From Fig. 2a-d, TB was the most sensitive index in the sites with cinnamon soil and fluvo-aquic soil. And the TB in the cinnamon soil

10 was much more sensitive than $\mathrm{HI}$ in fluvo-aquic soil. But as shown in Fig. $2 \mathrm{e}$ and $\mathrm{f}, \mathrm{HI}$ is the most sensitive parameter and sensitivity indices of BN3 and BP1 are much higher.

While using crop simulation models to stimulate crop growth at the regional scale, soil is crucial because it is responsible for a major part of the variability of crop model output variables of interest. The TB parameter controlled the optimal temperature. The

15 parameter's high sensitivity demonstrates that temperature is one of the most influencial factors for the crop growth in this study area. In brown soil, $\mathrm{HI}$ was the most sensitive parameter. $\mathrm{HI}$ is an important factor in calculating crop yield. It is adjusted by environmental stresses during the growing season. Higher sensitivity indices for $\mathrm{HI}$ mean equate to a decrease in environmental stress on crop growth. Clay concentration is much higher in brown soil, and brown soil is efficient at containing water and nutrients. The sites with brown soil are located in the region where temperature and precipitation is quite suitable for winter wheat. Water stress occurred less frequently in this region. The sensitivity indices of the parameters considering nutrition, such as BN3 and BP1, increased. Their sensitivity indices were lower than the parameter that relates

25 to LAl development in the other two soil types. Farm management was also an important factor in calculation of the sensitivity indices. Appropriate irrigation and fertilization can decrease water and nutrient stresses. It reduced the impact of parameters related to water and nutrients on crop growth and thus decreased their sensitivity indices. Due 3879

to less water stress and increased fertilization in this region, nutrient stress became more important to crop growth and made sensitivity indices of water and nutrient parameters increase.

\section{Conclusions}

5 Parameter sensitivity analysis is used to quantitatively apportion the variation of model output or different sources of variation and to find the parameters that are sensitive to model output. Conducting sensitivity analysis of the parameters at a regional scale and analyzing the differences of parameter sensitivity among stations would be much useful for model calibration and validation in different sub-regions. Overall this process

10 would benefit a model applied at the regional level. Through simulation of $2000 \times 22$ samples for 10 stations in the Huanghuaihai Plain, the rule of global sensitivity indices was found, and this study provides foundational work for model utilization in this region.

From the first order and the global sensitivity indices, TB (Optimal temperature), HI (Normal harvest index), WA (Potential radiation use efficiency), BN2 (Normal fraction 15 of $\mathrm{N}$ in crop biomass at mid-season) and RWPC1 (Fraction of root weight at emergency) were more sensitive than other parameters. These parameters influenced the crop yield directly. TB, which controlled the heat unit absorbed each day, was the determined parameter for crop assessment at the growing stage. WA controlled the energy absorbed, and $\mathrm{HI}$ calculated crop yield from the biomass.

Global sensitivity indices of parameters describing nutrients needed, such as BN1, $\mathrm{BN} 2$, and BN3, and the parameters related to LAI, such as DLAI, were much greater than their first-order sensitivity indices. This shows that some results in the crop growing process were highly dependent on these parameters. These parameters must be given attention during model calibration and validation. When using the crop simulation

25 model at the regional scale, soil was crucial because it was responsible for a major part of the variability of crop model output variables of interest. 
Acknowledgements. This work is supported by the National Key Technology R\&D Program (No. 2012BAH29B02-00).

\section{References}

Brun, R., Kuhni, M., Siegrist, H., Gujer, W., and Reichert, P.: Practical identifiablility of ASM2d parameters - systematic selection and turning of parameter subsets, Water Res., 36, 41134127, 2002.

Bryant, K. J., Benson, V. W., Kiniry, J. R., Williams, J. R., and Lacewell, R. D.: Simulating corn yield response to irrigation timings: validation of the EPIC model, J. Prod. Agric., 5, 237-242, 1992.

10 Drechsler, M.: Sensitivity analysis of complex models, Biol. Conserv., 86, 401-412, 1998.

Easterling, W. E., Weiss, A., Hays, C. J., and Mearns, L. O.: Spatial scales of climate information for simulating wheat and maize productivity: the case of the US Great Plains, Agr. Forest Meteorol., 90, 51-63, 1998.

Green, D. M. and Whittemore, C. T.: Calibration and sensitivity analysis of a model of the growing pig for weight gain and composition, Agr. Syst., 84, 279-295, 2005.

Griensven, A. v., Meixner, T., Grunwald, S., Bishop, T., Diluzio, M., and Srinivasan, R.: A global sensitivity analysis tool for the parameters of multi-variable catchment models, J. Hydrol., 324, 10-23, 2006.

Hasofer, A. M.: Modern sensitivity analysis of the CESARE-Risk computer fire model, Fire Safety J., 44, 330-338, 2009.

Janse, J. H., Scheffer, M., Lijklema, L., Liere, L. V., Sloot, J. S., and Mooij, W. M.: Estimating the critical phosphorus loading of shallow lakes with the ecosystem model PCLake: sensitivity, calibration and uncertainty, Ecol. Model., 221, 654-665, 2010.

Kang, S. Z., Zhang, L., Liang, Y. L., Hu, X., Cai, H., and Gu, B.: Effects of limited irrigation on yield and water use efficiency of water wheat in the Loess Plateau of China, Agr. Water Manage., 55, 203-216, 2002.

Klepper, O.: Multivariate aspects of model uncertainty analysis: tools for sensitivity analysis and calibration, Ecol. Model., 101, 1-13, 1997.

Lamboni, M., Makowski, D., Lehuger, S., Gabrielle, B., and Monod, H.: Multivariate global sensitivity analysis for dynamic crop models, Field Crop. Res., 113, 312-320, 2009.

Liu, J. G., Williams, J. R., and Alexander, J. B.: GEPIC-modeling wheat yield and crop water productivity with high resolution on a global scale, Agr. Syst., 94, 478-493, 2007.

Makowski, D., Naud, C., Jeuffroy, M.-H., Barbottin, A., and Monod, H.: Global sensitivity analysis for calculating the contribution of genetic parameters to the variance of crop model predicition, Reliab. Eng. Syst. Safe., 91, 1142-1147, 2006.

Norton, J. P.: Algebraic sensitivity analysis of environmental models, Environ. Modell. Softw., 23, 963-972, 2008.

Pappenberger, F., Beven, K. J., Ratto, M., and Matgen, P.: Multi-method global sensitivity analysis of flood inundation models, Advances in Water Resource, 31, 1-14, 2008.

10 Rinaldi, M.: Application of EPIC model for irrigation scheduling of sunflower in Southern Italy, Agr. Water Manage., 49, 185-196, 2001.

Saltelli, A., Tarantola, S., and Chan, K. P.: A quantitative model-independent method for global sensitivity analysis of model output, Technometrics, 41, 39-56, 1999.

Saltelli, A., Chan, K., and Scott, E. M.: Sensitivity Analysis, John Wiley and Sons, New York, 2000.

Satya, P. and Ryosuke, S.: National spatial crop yield simulation using GIS-based crop production model, Ecol. Model., 35, 113-129, 2001.

Tan, G. and Shibasaki, R.: Global estimation of crop productivity and the impacts of global warming by GIS and EPIC integration, Ecol. Model., 168, 357-370, 2003.

20 Varella, H., Guerif, M., and Buis, S.: Global sensitivity analysis measures the quality of parameter estimation: the case of soil parameters and a crop model, Environ. Modell. Softw., 25, 310-319, 2010.

Wallach, D., Goffinet, B., Bergez, J. E., Debaeke, P., Leenhardt, D., and Aubertot, J. N.: Parameter estimation for crop models: a new approach and application to a corn model, Agron. J., 25 93, 757-766, 2001.

Williams, J. R., Jones, C. A., Kiniry, J. R., and Spanel, D. A.: The EPIC crop growth model, T. ASAE, 32, 497-511, 1989.

Wu, J., Yu, F., Chen, Z., and Chen, J.: Global Sentivity analysis of growth simulation parameters of winter wheat based on EPIC model, T. CASE, 25, 136-142, 2009.

30 $\mathrm{Xu}, \mathrm{C}$. and Gertner, G.: Extending a global sensitivity analysis technique to models with correlated parameters, Comput. Stat. Data An., 51, 5579-5590, 2007.

$\mathrm{Xu}, \mathrm{C} ., \mathrm{Hu}, \mathrm{Y}$., Chang, Y., Jiang, Y., Li, X., Bu, L., and He, H.: Sensitivity analysis in ecological modeling, Chinese Journal of Applied Ecology, 15, 1056-1062, 2004. 
Table 1. Selected stations in this research area.

\begin{tabular}{ccclll}
\hline ID & Longitude & Latitude & Name & Province & Soil Type \\
\hline 53698 & 114.42 & 38.03 & Shijiazhuang & Hebei & Cinnamon soil \\
53798 & 114.50 & 37.07 & Xingtai & Hebei & Cinnamon soil \\
53986 & 113.88 & 35.32 & Xinxiang & Henan & Cinnamon soil \\
54618 & 116.55 & 38.08 & Botou & Hebei & Fluvo-aquic soil \\
54715 & 116.57 & 37.33 & Lingxian & Shandong & Fluvo-aquic soil \\
54725 & 117.53 & 37.48 & Huimin & Shandong & Fluvo-aquic soil \\
58005 & 115.67 & 34.45 & Shangqiu & Henan & Fluvo-aquic soil \\
54774 & 122.13 & 37.47 & Weihai & Shandong & Brown soil \\
54857 & 120.33 & 36.07 & Qingtao & Shandong & Brown soil \\
54945 & 119.53 & 35.43 & Rizhao & Shandong & Brown soil \\
\hline
\end{tabular}

Table 2. Crop parameters.

\begin{tabular}{llr}
\hline Parameter & Description & Initial Value \\
\hline WA & Potential radiation-use efficiency & 35.0 \\
HI & Normal harvest index & 0.42 \\
TB & Optimal temperature & 15.0 \\
DMLA & Maximum potential LAl (leaf area index) & 6.0 \\
DLAI & Point in the growing season when leaf area begins to decline due to leaf senescence & 0.6 \\
DLP1 & Crop parameter control leaf area growth of crop under non-stress control & 15.01 \\
DLP2 & Crop parameter control leaf area growth of crop under non-stress control & 50.95 \\
RLAD & LAl decline rate parameter & 0.4 \\
RBMD & Biomass-energy decline rate parameter & 0.4 \\
HMX & Maximum crop height & 0.4 \\
RDMX & Maximum root depth & 2.0 \\
WCY & Fraction of water in yield & 0.12 \\
BN1 & Normal fraction of N in crop biomass at emergence & 0.06 \\
BN2 & Normal fraction of N in crop biomass at mid-season & 0.023 \\
BN3 & Normal fraction of N in crop biomass at maturity & 0.0134 \\
BP1 & Normal fraction of $P$ in crop biomass at emergence & 0.0084 \\
BP2 & Normal fraction of $P$ in crop biomass at mid-season & 0.0032 \\
BP3 & Normal fraction of $P$ in crop biomass at maturity & 0.0019 \\
WAVP & The rate of decline in WA & 6.0 \\
RWPC1 & Fraction of root weight at emergence & 0.4 \\
RWPC2 & Fraction of root weight at maturity & 0.2 \\
GMHU & Accumulated heat units required for germination & 100.0 \\
\hline
\end{tabular}

"Mid-season is from emergence to flowering. 
Table 3. First-order sensitivity indices of the 22 parameters in the 10 selected stations.

\begin{tabular}{lcccccccccc}
\hline & Shijiazhuang & Xingtai & Xinxiang & Botou & Lingxian & Huimin & Shangqiu & Weihai & Qingtao & Rizhao \\
\hline WA & 0.1191 & 0.1382 & 0.1275 & 0.0920 & 0.0750 & 0.0778 & 0.0938 & 0.1729 & 0.1586 & 0.1598 \\
HI & 0.2458 & 0.2643 & 0.2391 & 0.1468 & 0.1185 & 0.1226 & 0.1980 & 0.3190 & 0.5034 & 0.4367 \\
TB & 0.3675 & 0.3044 & 0.3554 & 0.5009 & 0.5749 & 0.5619 & 0.4094 & 0.1464 & 0.0223 & 0.0534 \\
DMLA & 0.0059 & 0.0062 & 0.0057 & 0.0061 & 0.0051 & 0.0055 & 0.0053 & 0.0053 & 0.0060 & 0.0062 \\
DLAl & 0.0215 & 0.0255 & 0.0305 & 0.0192 & 0.0220 & 0.0215 & 0.0281 & 0.0255 & 0.0324 & 0.0302 \\
DLP1 & 0.0154 & 0.0177 & 0.0139 & 0.0145 & 0.0120 & 0.0104 & 0.0101 & 0.0054 & 0.0073 & 0.0088 \\
DLP2 & 0.0268 & 0.0308 & 0.0345 & 0.0284 & 0.0267 & 0.0263 & 0.0184 & 0.0193 & 0.0294 & 0.0293 \\
RLAD & 0.0033 & 0.0036 & 0.0034 & 0.0027 & 0.0040 & 0.0047 & 0.0029 & 0.0027 & 0.0042 & 0.0040 \\
RBMD & 0.0051 & 0.0074 & 0.0040 & 0.0072 & 0.0035 & 0.0059 & 0.0033 & 0.0036 & 0.0021 & 0.0033 \\
HMX & 0.0044 & 0.0045 & 0.0022 & 0.0040 & 0.0022 & 0.0024 & 0.0043 & 0.0029 & 0.0009 & 0.0013 \\
RDMX & 0.0063 & 0.0061 & 0.0077 & 0.0066 & 0.0072 & 0.0075 & 0.0096 & 0.0065 & 0.0067 & 0.0063 \\
WCY & 0.0002 & 0.0003 & 0.0003 & 0.0001 & 0.0002 & 0.0003 & 0.0002 & 0.0005 & 0.0003 & 0.0005 \\
BN1 & 0.0032 & 0.0028 & 0.0050 & 0.0020 & 0.0033 & 0.0027 & 0.0029 & 0.0061 & 0.0051 & 0.0056 \\
BN2 & 0.0468 & 0.0357 & 0.0241 & 0.0282 & 0.0195 & 0.0219 & 0.0477 & 0.0809 & 0.0626 & 0.0613 \\
BN3 & 0.0054 & 0.0059 & 0.0031 & 0.0018 & 0.0010 & 0.0017 & 0.0094 & 0.0577 & 0.0181 & 0.0177 \\
BP1 & 0.0034 & 0.0044 & 0.0079 & 0.0020 & 0.0045 & 0.0030 & 0.0050 & 0.0065 & 0.0066 & 0.0067 \\
BP2 & 0.0041 & 0.0031 & 0.0051 & 0.0055 & 0.0070 & 0.0067 & 0.0041 & 0.0026 & 0.0007 & 0.0013 \\
BP3 & 0.0058 & 0.0060 & 0.0063 & 0.0053 & 0.0052 & 0.0050 & 0.0061 & 0.0055 & 0.0053 & 0.0049 \\
WAVP & 0.0007 & 0.0012 & 0.0008 & 0.0004 & 0.0004 & 0.0006 & 0.0011 & 0.0026 & 0.0047 & 0.0026 \\
RWPC1 & 0.0234 & 0.0257 & 0.0216 & 0.0152 & 0.0123 & 0.0120 & 0.0195 & 0.0422 & 0.0513 & 0.0405 \\
RWPC2 & 0.0193 & 0.0291 & 0.0242 & 0.0154 & 0.0105 & 0.0120 & 0.0238 & 0.0263 & 0.0308 & 0.0382 \\
\hline
\end{tabular}

3885

Table 4. Global sensitivity indices of 22 parameters in the 10 selected stations.

\begin{tabular}{lcccccccccc}
\hline & Shijiazhuang & Xingtai & Xinxiang & Botou & Lingxian & Huimin & Shangqiu & Weihai & Qingtao & Rizhao \\
\hline WA & 0.1576 & 0.1696 & 0.1606 & 0.1257 & 0.1078 & 0.1144 & 0.1249 & 0.2052 & 0.2412 & 0.2277 \\
HI & 0.2665 & 0.2908 & 0.2622 & 0.1655 & 0.1406 & 0.1455 & 0.2187 & 0.3606 & 0.7250 & 0.5898 \\
TB & 0.4682 & 0.4021 & 0.4425 & 0.5933 & 0.6600 & 0.6529 & 0.4947 & 0.2326 & 0.0813 & 0.1286 \\
DMLA & 0.0402 & 0.0373 & 0.0294 & 0.0348 & 0.0320 & 0.0330 & 0.0305 & 0.0318 & 0.0259 & 0.0297 \\
DLAl & 0.0582 & 0.0584 & 0.0658 & 0.0467 & 0.0552 & 0.0508 & 0.0614 & 0.0561 & 0.0756 & 0.0689 \\
DLP1 & 0.0730 & 0.0785 & 0.0774 & 0.0592 & 0.0706 & 0.0665 & 0.0651 & 0.0333 & 0.0318 & 0.0414 \\
DLP2 & 0.0480 & 0.0519 & 0.0564 & 0.0464 & 0.0475 & 0.0467 & 0.0374 & 0.0445 & 0.0656 & 0.0599 \\
RLAD & 0.0309 & 0.0236 & 0.0229 & 0.0303 & 0.0361 & 0.0367 & 0.0216 & 0.0137 & 0.0197 & 0.0154 \\
RBMD & 0.0137 & 0.0269 & 0.0151 & 0.0323 & 0.0111 & 0.0163 & 0.0396 & 0.0195 & 0.0110 & 0.0151 \\
HMX & 0.0174 & 0.0226 & 0.0129 & 0.0151 & 0.0097 & 0.0094 & 0.0271 & 0.0171 & 0.0083 & 0.0107 \\
RDMX & 0.0467 & 0.0416 & 0.0521 & 0.0437 & 0.0536 & 0.0472 & 0.0629 & 0.0497 & 0.0639 & 0.0602 \\
WCY & 0.0025 & 0.0029 & 0.0024 & 0.0022 & 0.0021 & 0.0024 & 0.0026 & 0.0036 & 0.0075 & 0.0056 \\
BN1 & 0.0222 & 0.0293 & 0.0341 & 0.0164 & 0.0217 & 0.0182 & 0.0358 & 0.0460 & 0.0560 & 0.0535 \\
BN2 & 0.0783 & 0.0790 & 0.0880 & 0.0691 & 0.0857 & 0.0771 & 0.1097 & 0.1283 & 0.1383 & 0.1292 \\
BN3 & 0.0201 & 0.0407 & 0.0156 & 0.0215 & 0.0111 & 0.0169 & 0.0365 & 0.1198 & 0.0694 & 0.0781 \\
BP1 & 0.0224 & 0.0453 & 0.0532 & 0.0198 & 0.0249 & 0.0210 & 0.0503 & 0.0700 & 0.0706 & 0.0802 \\
BP2 & 0.0266 & 0.0203 & 0.0283 & 0.0361 & 0.0498 & 0.0429 & 0.0431 & 0.0160 & 0.0058 & 0.0081 \\
BP3 & 0.0480 & 0.0476 & 0.0524 & 0.0408 & 0.0475 & 0.0434 & 0.0507 & 0.0467 & 0.0522 & 0.0512 \\
WAVP & 0.0074 & 0.0081 & 0.0101 & 0.0086 & 0.0130 & 0.0122 & 0.0081 & 0.0103 & 0.0244 & 0.0155 \\
RWPC1 & 0.0765 & 0.0847 & 0.0858 & 0.0579 & 0.0589 & 0.0596 & 0.0735 & 0.1175 & 0.1448 & 0.1257 \\
RWPC2 & 0.0319 & 0.0582 & 0.0414 & 0.0352 & 0.0202 & 0.0248 & 0.0510 & 0.0664 & 0.1289 & 0.1293 \\
GMHU & 0.0591 & 0.0637 & 0.0487 & 0.0492 & 0.0110 & 0.0207 & 0.0532 & 0.0829 & 0.0638 & 0.0678 \\
\hline
\end{tabular}




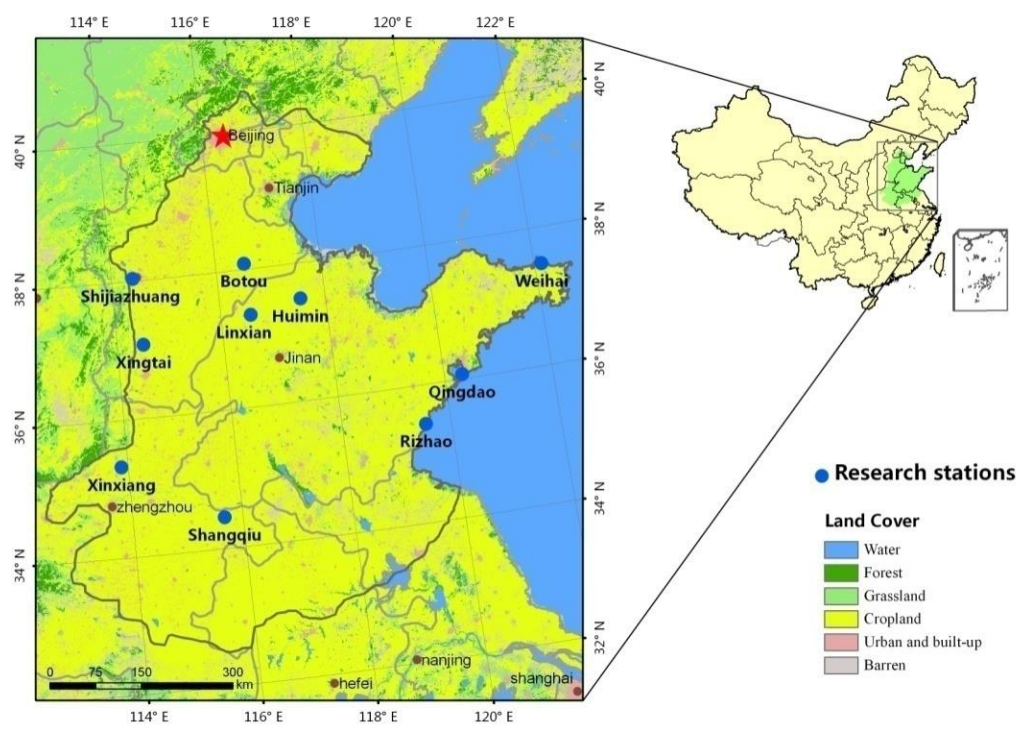

Figure 1. Study sites located in the Huanghuaihai Plain.
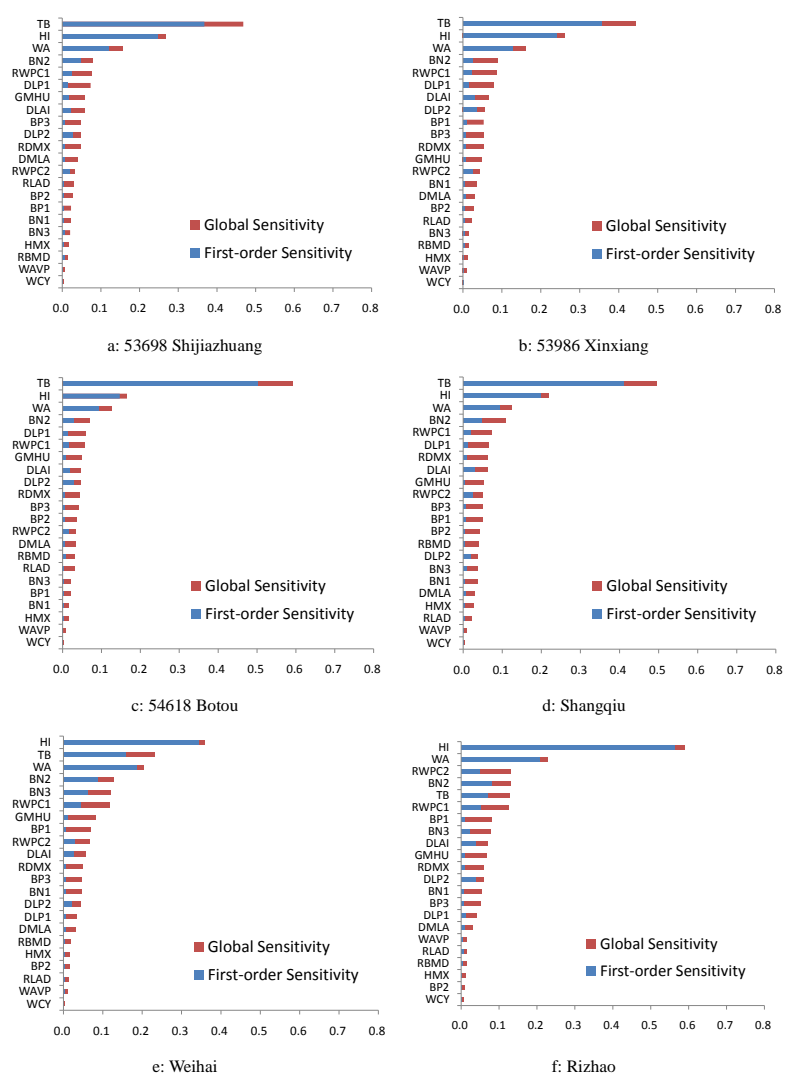

Figure 2. Sensitivity indices in 6 sites. 